\title{
Application of Nano Rare Earth Materials
}

\author{
Hong $\mathrm{Li}^{1, *}$, Fengjun $\mathrm{Wei}^{1}$, Ruixiao $\mathrm{Gao}^{1}$ \\ ${ }^{1}$ Shandong Huayu University of Technology, Dezhou, Shandong, China.
}

\begin{abstract}
Rare earth is an important strategic resource in China, and it has the reputation of "industrial vitamin" in the industrial field. Rare earth is non-renewable. Due to the diverse preparation methods of rare earth oxides, different preparation methods have their own advantages and disadvantages. Using different methods to prepare nano-rare earth oxides. And because of the special electronic structure of rare earth elements, it has special optical, electrical and magnetic properties. This article mainly introduces the use of nano-rare earth oxides in luminescence, permanent magnetism, ceramics, catalysis and so on.
\end{abstract}

\section{Introduction}

The special electronic configuration of rare earth elements gives them special optical, electrical and magnetic properties, and is known as a treasure house of new materials. Rare earth oxides are an important type of rare earth compounds and have a wide range of uses, while nano-rare earth oxides can exhibit more superior performance. This article reviews the preparation methods and applications of nano-rare earth oxides, and introduces the applications of rare earth compounds in different industries.

Rare earth elements themselves have a rich electronic structure, showing many optical, electrical, and magnetic properties. After nanometerization, they have many new properties, such as small size effect, high surface area effect, quantum effect, and extremely strong photoelectromagnetic and acoustic properties. Superconductivity, high chemical activity, etc., can greatly improve the comprehensive performance of materials, develop many new functional materials, in optical materials, luminescent materials, crystal materials, magnetic materials, battery materials, electronic ceramics, engineering ceramics, engineering plastics, packaging Materials, precision polishing materials and other fields have a wide range of applications and play an important role.

My country's rare earth exports account for more than $70 \%$ of the world's rare earth consumption and have an absolute market advantage. However, most of them are exported as raw materials of chemical and mineral products, which are of low value and waste resources. The application of rare earth nanomaterials in electronic high-tech products lags behind developed countries such as Japan and the United States. Therefore, the application of rare earth nanomaterials in electronic materials is vigorously promoted to effectively utilize my country's rich rare earth resources, increase the value of rare earth resources, and increase export earnings. Ability has important practical significance.

\section{Preparation method of nano rare earth oxide}

Rare earth elements have a special electronic layer structure and a large atomic radius, and their chemical properties are very different from other elements. Therefore, the preparation method and post-processing technology of nano-rare earth oxide powder are also different from other elements. In summary, the main synthesis methods of nano- $\mathrm{CeO}_{2}[1-3]$ include precipitation method, hydrothermal method, gel method, solid phase method, organic microemulsion method, alkoxide hydrolysis method, etc. The characteristics of several methods are as follows:

1) Precipitation method: The preparation of nano-rare earth oxides by precipitation method is to add a precipitation agent to the rare earth salt solution, or hydrolyze the solution at a certain temperature to form insoluble hydroxides, hydrated oxides or salts to precipitate out of the solution, and remove the solvent from the solution. And the original ions in the solution are washed away, and the required oxide powder is obtained by roasting and decomposing or dehydrating[4]. Precipitation methods include oxalic acid precipitation, carbonic acid precipitation, hydroxide precipitation, homogeneous precipitation, complex precipitation and so on. The precipitation method is the most commonly used method for research and industrial production. This method has the advantages of low raw material cost, low equipment requirements, simple process, simple operation, accurate chemical composition control, and easy preparation of ultrafine powders with uniform components. It is easy to control the shape and size of the

\footnotetext{
* Corresponding author: 1hong25@163.com
} 
particles, and can produce high-purity products, but it is difficult to filter and easy to agglomerate[5].

2)Hydrothermal method: Hydrothermal method refers to the use of water or alcohol-water mixed solution in a highly sealed autoclave as a solvent, and the autoclave is heated at a certain temperature to form a high temperature and high pressure environment. At this time, it is insoluble or insoluble under normal conditions. The reactants dissolve and react, and then, driven by the temperature difference of the solution in the autoclave, strong convection is generated to form a supersaturated solution and then crystallize out[6]. The method can obtain nano powders with uniform dispersion, narrow particle size distribution, good crystal shape and controllable appearance, but requires high temperature and high pressure equipment, expensive equipment, low efficiency, and unsafe operation.

3)Sol-gel method: It is an important method for preparing inorganic materials and occupies a considerable position in inorganic synthesis. At low temperatures, organometallic compounds or organic complexes, through polymerization or hydrolysis, etc., form a sol, under certain conditions, form a gel, and further heat treatment. That is, through the preparation of the homogeneous solution-the preparation of the sol-the gelation process-the drying of the gel-the heat treatment process of the xerogel, the ultrafine nanopowder with larger specific surface and better dispersion can be finally obtained. The method can be carried out under mild conditions, and the powder obtained is larger than the surface and has good dispersibility, but the reaction time is longer, it takes several days to complete, and it is difficult to meet the requirements of large-scale industrialization[7].

4)Solid phase method: High temperature decomposition is carried out by solid compound or intermediate solid phase reaction. For example, the rare earth salt is mixed with oxalic acid or ammonium bicarbonate in solid phase to form an intermediate of the rare earth salt, which is then decomposed at high temperature to obtain ultrafine powder. The solid phase method can be roughly divided into two categories: mechanical crushing method and solid phase reaction method. The former is that the material is crushed into finer particles under the action of machinery. This method is easy to operate and has a relatively low cost, but the resulting product has poor uniformity and it is difficult to obtain ultrafine particles. The latter refers to a method in which the reactants are proportioned in a certain proportion and subjected to a grinding and calcination process to obtain ultra-fine powder[6]. In summary, the solid-phase method for preparing rare earth oxides has high reaction efficiency, simple equipment and easy operation, but the obtained powder has irregular morphology and poor uniformity.

\section{Application of Nano Rare Earth Materials}

\subsection{Application of Nano Rare Earth Catalytic Technology in Automobile Exhaust Gas Purification}

Automobile exhaust is one of the main sources of air pollution today. Catalytic purification is a method of purifying automobile exhaust that is commonly used internationally. Nano-rare earth catalyst is a new type of high-efficiency automobile exhaust purification catalyst prepared by combining the high surface activity of nano-materials with the characteristics of rare earth catalytic promoters in the catalyst. This catalyst combines the advantages of nano-materials and rare earths. Can effectively purify car exhaust[8].

At present, most of the ternary automobile exhaust catalytic converters containing precious metals are used for external purification of automobile exhausts, it can convert nitrogen oxides and carbon oxides in the exhaust gas into nitrogen, carbon dioxide, water, etc., but precious metals are expensive and are prone to $\mathrm{Pb}, \mathrm{S}, \mathrm{P}$ poisoning. Rare earth nano materials integrate the characteristics of rare earth and nano materials. Using nano rare earth particles to replace conventional rare earth compounds in the three-way catalyst can increase the conversion rate of $\mathrm{CO}, \mathrm{NO}_{\mathrm{X}}$ and $\mathrm{CH}$ in automobile exhaust. Studies have shown[9] that composite rare earth compound nanopowders have extremely strong redox performance, which can completely solve the pollution problems of carbon monoxide $(\mathrm{CO})$ and nitrogen oxides $\left(\mathrm{NO}_{\mathrm{X}}\right)$ in automobile exhaust.

\subsection{Application of nano-rare earth in electronic materials}

Rare earth nano-powder modified epoxy resin has greatly improved high temperature resistance, toughness, strength, etc., in the application of electronic circuit substrates and packaging materials, the overall performance has been significantly improved and improved, and the amount is small, and the price is low. Long life and other advantages, foreign countries use hundreds of tons per year, but our country has almost no industrial application, the reason is likely to be technical secrecy. Used in the modification of organic silicon materials, it can improve its high temperature resistance and anti-ultraviolet, anti-aging, anti-radiation, etc., and can better meet the special requirements of electronic and electrical appliances for organic silicon materials. The YBCO superconductor prepared by using nanometer $\mathrm{Y}_{2} \mathrm{O}_{3}$ is a special thin film material with stable performance, high strength, easy processing, close to the practical stage, and broad prospects.

\subsection{Application of rare earths in luminescent materials}

Rare earth luminescent nanomaterials are a new type of 
material with stable chemical and physical properties, can withstand the effects of high-power electron beams, high temperature resistance, strong ultraviolet light and high-energy radiation, and have bright colors, high color purity, and narrow emission bands; high conversion efficiency, strong light absorption; fluorescence lifetime can reach nanometer level; wide emission wavelength distribution area[10-11].

Rare earth luminescent materials have a very wide range of uses. They can be used not only in metallurgy and glass production in the industrial field, but also in the production of medical imaging, radar or computer display screens, and also in the production of daily lighting fixtures[12]. The application of rare earth ion luminescent materials in the fields of biomedicine and energy. Rare earth ions are used in high-energy ion detectors. Related materials are called scintillators. These scintillators have a wide range of applications in the detection of cosmic rays in biomedical diagnosis. The scintillator requires fast response, high luminous efficiency, good linearity of signal response and high density[13]. Wang et al. synthesized red-emitting $\mathrm{YVO}_{4}: \mathrm{Eu}$ nanocrystals and green-emitting $\mathrm{LaPO}_{4}: \mathrm{Ce}, \mathrm{Tb}$ nanoribbons, and used them as fluorescent labels in the field of fingerprint forensics. Compared with commonly used materials, they have high sensitivity. The advantages of high contrast, high efficiency and low background interference[14].

\subsection{Application of nano-rare earth in high-performance ceramics}

Electronic ceramics (electronic sensors, PTC materials, microwave materials, capacitors, thermistors, etc.). Prepared using ultra-fine or nano-level $\mathrm{Y}_{2} \mathrm{O}_{3}, \mathrm{La}_{2} \mathrm{O}_{3}$, $\mathrm{Nd}_{2} \mathrm{O}_{3}, \mathrm{Sm}_{2} \mathrm{O}_{3}$, etc., have many improvements in electrical performance, thermal performance, and stability. Important aspects of electronic materials upgrade. For example, ceramics sintered with nano $\mathrm{Y}_{2} \mathrm{O}_{3}$ and $\mathrm{ZrO}_{2}$ at a lower temperature have high strength and toughness and are used in wear-resistant devices such as bearings and tools; multilayer chip capacitors and microwave devices made of nano $\mathrm{Y}_{2} \mathrm{O}_{3}, \mathrm{Nd}_{2} \mathrm{O}_{3}, \mathrm{Sm}_{2} \mathrm{O}_{3}$, etc., performance is greatly improved[15-16]. Xie Min et al. used rare earth ion $\mathrm{Y} 3+$ to replace $\mathrm{Sm} 3+$, which made the grain size of $\mathrm{Sm}_{2-\mathrm{x}} \mathrm{Y}_{\mathrm{x}} \mathrm{ZrO}_{7}$ ceramic material smaller, the grain size of the ceramic material was reduced, the density was reduced, and the sintering resistance was enhanced[17].

\subsection{Application of Nano Rare Earth in Precision Polishing}

$\mathrm{CeO}_{2}$ has a better polishing effect on glass, while nano- $\mathrm{CeO}_{2}$ has a higher polishing precision. It has been used in liquid crystal displays, silicon single wafers, glass storage, etc. At present, the new generation of CMP polishing liquids used in the US, Japan, South Korea, etc., for semiconductor silicon wafers and ULSI leveling, all contain nano- $\mathrm{CeO}_{2}$. The literature shows that the polishing efficiency of using nano- $\mathrm{CeO}_{2}$ polishing agent is more than 2.7 times higher than that of silica sol polishing agent. Therefore, nano- $\mathrm{CeO}_{2}$ will gradually replace silica sol[18]. The cerium oxide precision polishing powder prepared by Liu Zhaogang and others by the precipitation method has a cubic crystal structure and a $\mathrm{CaF}_{2}$ fluorite structure, with a relatively uniform particle size distribution and good particle dispersion. The $\mathrm{D}_{50}$ is $0.149 \mu \mathrm{m}$ and the specific surface area is 31.05 $\mathrm{m}^{2} / \mathrm{g}$, suitable for polishing in high-tech fields.

\subsection{Application of Nano Rare Earth in Magnetic Materials}

Nano-rare-earth magnetic materials can be used in magnetic storage, magnetic fluid, giant magnetoresistance, etc., with greatly improved performance, making the device high-performance and small. For example, the giant magnetoresistance target $\mathrm{REMnO}_{3}$ prepared from nano-rare earth oxides has great value in the application of giant magnetoresistance films; adding nano-Ceria to ferrite magnets can greatly improve its magnetic properties. In short, the application of rare earth nanomaterials in the electronics field has just begun, with remarkable functions, wide application, low cost, huge potential, and very promising commercial prospects, and it is worthy of vigorous promotion and application[19].

\subsection{Application of Nano Rare Earth in Solid Fuel Cells}

The energy crisis in the 21 st century is becoming more and more serious. All countries are committed to the development of efficient and clean energy systems. Fuel cells came into being, which is of great significance. As an energy storage device with high energy and strong stability, the emergence of fuel cells provides an effective way to achieve sustainable energy development. The fuel cell technology has gradually developed to today's solid oxide fuel cell (SOFC) through continuous updating. SOFC is in vigorous development. It is called the third-generation fuel cell. It has an all-solid-state structure and can directly convert chemical energy into electric energy under high temperature conditions. It has a large selection of raw materials, high energy conversion rate, and low cost. Many advantages such as low pollution. Doped cerium oxide has high ionic conductivity, and a series of nano-functional materials can be prepared by selecting appropriate components for doping, making full use of the redox performance of $\mathrm{Ce}^{3+} / \mathrm{Ce}^{4+}$ in the two groups of compounds, and increasing the temperature of the fuel cell. Output performance[20].

\section{Conclusion}

My country is rich in rare earth minerals, and its mining and smelting technology is relatively advanced, which provides a reliable resource guarantee for the progress of my country's modern industry. Currently, different methods for preparing nano-rare earth materials have been developed, and significant results have been achieved. Rare earth nanomaterials are a new type of 
material with stable chemical properties and good application prospects. They play a huge role in promoting the development of modern society and improving people's quality of life. At the same time, compared with foreign developed countries, my country still has a big gap in technology research and development and efficient utilization of rare earth resources. This requires that my country's research on rare earth luminescent materials related technology needs to be further strengthened, and strive to maximize the use of rare earth resources, so that it can bring greater convenience to the production and life of our people.

\section{Acknowledgments}

Thanks for the support of the first-class specialty construction fund of Shandong Huayu University of Technology.

Thank you for the support of the Dezhou Key Laboratory of High-efficiency Heat Pump Air Conditioning Equipment and System Energy Saving Technology of Shandong Huayu University of Technology.

\section{References}

1. Guo Wenting. Research on Synthetic Method and Application of Rare Earth Oxide Nanomaterials[J]. Education Teaching Forum, 2014(31):120-121.

2. Huo Jianzhen, Wei Mingzhen. Preparation and Application of Nano Rare Earth Oxides[J]. Sichuan Chemical Industry, 2006(02):26-29.

3. W.-J. Kim,J. Y. Park,S. J. Oh,Y. S. Kim,G.-W. Hong,I.-H. Kuk. Characteristics and Sintering Behavior of Yttria Powders Synthesized by the Combustion Process[J]. Journal of Materials Science Letters, 1999,18(5).

4. Wang Xiaolan, Li Lili, Duan Xuechen. Preparation of Nanometer Yttrium Oxide Powder[J]. Rare Metals and Cemented Carbides, 2004(01): 26-28+35.

5. Xiong Xiao-bai, Liu Ling-sheng, Li Ming-dong, Jia Tao, Ma Sheng-feng, Hu Wei-hong. Status in Study of Nanometer Rare Earth Oxides Prepared by Precipitation Methods[J]. Chinese Rare Earths, 2013,34(02):81-86.

6. Wang Zhuhong. Hydrothermal Preparation and Performance Research of Cerium Oxide Micro-nano Materials[D]. Nanjing University of Science and Technology, 2012.

7. Hou Wenhua, Xu Lin, Qiu Jinheng, Guo Canxiong, Chen Ligang, Yan Qijie. Preparation of Ultrafine Ceric Oxide Particles by Different Methods-I. Sol-Gel Method[J]. Journal of Nanjing University (Natural Sciences), 1999(04):486-490.

8. Xiang-Huiqinag, Liu-Jianyong, Tan-Lili. Application of Nanocatalytia Techniques of Rare Earths for Purification of Auto-mobile Exhaust Gases.[J]. Modern Chemical Industry, 2006(S2):379-381.
9. Shen Dixin, Chen Hongde, Tian Qun. Automobile Exhaust Pollution, Emission Control and Strategies in China[J]. Progress in Environmental Science, 1997(06):24-34.

10. Xing Yunhu, Mao Weixing, Liu Lixing. Analysis on Development and Application of Rare Earth Luminescent Materials[J]. Liaoning Chemical Industry, 2020,49(12):1561-1562+1580.

11. Wang Shutin, Yang Shuting. Luminescence Mechanism and Application of Rare Earth Luminescent Materials[J]. Technology Outlook, 2015, 25 (36): 132.

12. .Liu Dongyang. Analysis on the Luminescence Mechanism and Application of Rare Earth Luminescent Materials[J]. Chemical Management, 2019(31):47-48.

13. Jiang Yajiang, Wang Qiaomei. Application of Nano Rare Earth in Electronic Materials[A]. China Electronic Materials Industry Association. Proceedings of Symposium on High-tech and Market of Electronic Specialty Chemical[C]. China Electronic Materials Industry Association: Economic and Technical Management Department of China Electronic Materials Industry Association, 2004:4.

14. Wang M, Li M, Yu A Y, et al. Uniform lanthanide-doped $\mathrm{Y} 2 \mathrm{O} 3$ hollow microspheres: Controlled synthesis and luminescence properties[J]. Materials Science and Engineering B, 2011,176:1251-1256.

15. New Technology for Preparing Rare Earth-Dopedyttrium Aluminum Garnet HighPerformance Ceramic Powder. Shandong Provice, Qilu University of Technology, 2016-01-01.

16. Yin Yue, Ma Beiyue, Li Ying, Yu Jingkun. New Progress in the Application of Rare Earth Oxides in Ceramic Materials[A]. 2015 Refractory Comprehensive Academic Annual Conference. (The $13^{\text {th }}$ National Academic Conference on Unshaped Refractories and 2015Refractory Materials Academic Exchange Coference) Proceedings (1) [C]. Wuhan University of Science and Technology Jointly Established the State Key Laboratory of Refractories and Metallurgy: Chinese Society of Metals, 2015:8.

17. Xie Min, Song Xiwen, Zhou Fen, An Shengli. Study on Structure and Thermo-Physical Properties of $\mathrm{Sm}_{2-\mathrm{x}} \mathrm{Y}_{\mathrm{x}} \mathrm{ZrO}_{7}$ Ceramics for Thermal Barrier Coatings[J]. Functional Materials, 2015,46(24):24038-24042.

18. Liu Zhaogang, Li Mei, Hu Yanhong, Guo Ruihua, Wang Mitang. The Preparation of Cerium Oxide Ultrafine Polishing Powder by Ammonia-Oxalic Acid Coprecipitation[A]. Professional Committee of Particle Preparation and Processing of Chinese Society of Particles. Symposium on Recent Progress in Particuology-10 ${ }^{\text {th }}$ National Conference on Particle Preparation and Treatment[C]. Professional Committee of Particle Preparation and Processing of Chinese Society of Particles: Chinese Society of Particles, 2011:5. 
19. Nababan Deddy C.,Mukhlis Reiza,Durandet Yvonne,Pownceby Mark I.,Prentice Leon,Rhamdhani M Akbar. Mechanism and microstructure evolution of high temperature oxidation of end-of-life $\mathrm{NdFeB}$ rare earth permanent magnets $[\mathrm{J}]$. Corrosion Science,2021,182.

20. Milliken C E, Guruswamy S. Electrochemical Stability of Strontium-doped Ceria Electrolyte in Solid-oxide Fuel Cell Applications. J. Am. Ceram. Soc., 2001, 84(7):1533-1538. 